\title{
THE BOARDMAN CATEGORY OF SPECTRA, CHAIN COMPLEXES AND (CO-)LOCALIZATIONS
}

\author{
FRIEDRICH W. BAUER \\ submitted by Walter Tholen
}

\begin{abstract}
This is a survey of the author's results about chain functors, tensor products of chain functors, (co-)localizations of chain functors and their relationship with the associated concepts for spectra in the Boardman category. Some introductory remarks about quotient categories, stable homotopy theory and application of the theory to strong homology theories are included.
\end{abstract}

\section{Introduction}

This is an expository article about the author's recent work on a specific aspect of the theory of chain functors [8], their relations with Boardman spectra, tensor products and the theory of (co-)localizations, expressed by means of chain functors [3], [4].

The Boardman category $\mathfrak{B}$ appeared as the first appropriate category in which one can satisfactorily develop stable homotopy theory. All other stable homotopy categories should be equivalent to this category.

A spectrum $E$ determines a homology theory $E_{*}()$ which originates from a chain functor $\boldsymbol{C}_{*}=\boldsymbol{C}_{*}(E)(\mathrm{cf} . \S 3)$ in the sense that the associated homology theory $H_{*}\left(\boldsymbol{C}_{*}\right)(\mathrm{)})$ of $\boldsymbol{C}_{*}$ is isomorphic to $E_{*}()$.

However every chain functor $\boldsymbol{K}_{*}$, defined on a category of CW pairs with compact support can be geometrically realized as a spectrum $F=\left|\boldsymbol{K}_{*}\right|$ such that $F_{*}() \approx H_{*}\left(\boldsymbol{K}_{*}\right)()$.

So we have a cycle of relationships:

$$
E \in \mathfrak{B} \longmapsto E_{*}() \longmapsto\left|C_{*}(E)\right| \simeq E .
$$

In the category of chain functors $\mathfrak{C h}$, cf. $\S 3$, there are tensor products mimicked after the concept of a tensor product between chain complexes. The cycle (1) provides us with the opportunity to define tensor products between spectra (cf. §4) by setting

$$
E \otimes F \approx\left|\boldsymbol{C}_{*}(E) \otimes \boldsymbol{C}_{*}(F)\right| .
$$

This tensor product cannot be defined directly for spectra, avoiding chain functors.

$\mathrm{H}$. Hasse always pointed out, that all number theory (including analytic number theory) is ultimately designed to furnish results about integers. In the same sense I think that all results about chain functors should be assessed by the amount of information they yield for topological spaces, eventually for spectra and their related homology theories in the Boardman category.

One has a satisfactory theory of localizations of chain functors and a dual theory of colocalizations, leading to existence (and by definition, uniqueness ) theorems for localizations and colocalizations $(\S 5, \S 6)$.

This distinguishes chain functors from spectra: Although according to A. K. Bousfield [12] there exists always an E-localization, not every spectrum admits an E-colocalization. The

Received 22 April 1999, revised 11 May 1999; published on 17 May 1999.

1991 Mathematics Subject Classification: Primary 55P60, 55P42, 55N20; Secondary 55U15, 55N07

Key words and phrases: chain functors; Boardman spectra; Tensor products; localizations; colocalizations

(C) 1999, Friedrich W. Bauer. Permission to copy for private use granted. 
reason is that the $E$-colocalization of a chain functor $\boldsymbol{A}_{*}$ (with compact carriers) does not necessarily has compact carriers itself, hence it cannot be realized as a spectrum $\left|\boldsymbol{A}_{*}^{E}\right|$. However if one starts with a homology theory without compact carriers like

$$
h_{*}()=\{E,\}_{*},
$$

detect the chain functor associated with $h_{*}$, apply the colocalization process to $C_{*}=C_{*}(F)$, the chain functor associated with another spectrum $F$, then the resulting chain functor $C_{*}^{[E,]}$ has compact carriers, admitting a geometric realization $F^{[E,]}$ (cf. $\S 6$ ). This is in accordance with a result of A. K. Bousfield [13].

There is a surprising relationship between tensor products of chain functors and localizations $(\S 5)$ :

In $\S 3$ we introduce a highly irregular chain functor $\mathbb{Z}_{*}$, (which cannot be realized as a spectrum and whose derived homology is not a homology theory) serving as a unit for tensor products of chain functors. One has for any chain functor $\boldsymbol{K}_{*}$ an equivalence of irregular chain functors

$$
K_{*} \otimes \mathbb{Z}_{*} \approx K_{*} .
$$

It turns out that for any $E$-localization $\boldsymbol{A}_{E *}$ of a chain functor $\boldsymbol{A}_{*}$ one has

$$
\boldsymbol{A}_{*} \otimes \mathbb{Z}_{E *} \simeq \boldsymbol{A}_{E *} .
$$

In our previous exposition we have tacitly assumed that all spaces involved are $\mathrm{CW}$ spaces or ANRs. However chain functors were originally introduced for a description of strong homology theories ( $\S 7$ ), thus they can be defined and used for much more general spaces (or spectra). Exploiting this yields a characterization of strong homology theories as associated homology theories of localizations of chain functors with respect to the category of "good spaces" (like CW spaces) (theorem 7.1.).

In $\S 1$ we recollect some elementary facts about quotient categories and define (co-)localizations in a very general framework.

While $\S 2$ contains some well-known facts about stable homotopy and spectra, $\S 3$ serves as an introduction for chain functors.

With the exception of theorem 3.3. (which is proved in $\S 8$, because this proof serves simultaneously as an explicit example of a chain functor) this paper does not contain proofs; the reader is referred to the original sources in the literature. 


\section{Quotient categories, (co-)localizations}

Let $\mathfrak{K}$ be a category, $\mathfrak{M} \subset \mathfrak{K}$ a class of morphisms, then the quotient category $(\mathfrak{K} / \mathfrak{M}, \eta)$ consists of a category $\mathfrak{K} / \mathfrak{M}$ and a functor $\eta: \mathfrak{K} \longrightarrow \mathfrak{K} / \mathfrak{M}$ which is universal with respect to the condition that $\eta(m)$ is an isomorphism for any $m \in \mathfrak{M}$. More precisely:

Let $\rho: \mathfrak{K} \longrightarrow \mathfrak{L}$ be any functor, satisfying $\rho(m)=$ isomorphism for any $m \in \mathfrak{M}$, then there exists a unique functor $\bar{\rho}: \mathfrak{K} / \mathfrak{M} \longrightarrow \mathfrak{L}$ such that $\bar{\rho} \eta=\rho$.

An existence proof is given in [11]. In [18] the authors call this a category of fractions.

There are many different classes $\mathfrak{M}$ leading to the same category $\mathfrak{K} / \mathfrak{M}$ : If $\mathfrak{M}_{1}=\{$ identities $\}$, $\mathfrak{M}_{2}=\{$ isomorphisms $\}, \mathfrak{M}_{3}=\emptyset$, then $\mathfrak{K} / \mathfrak{M}_{i}$ is always isomorphic to $\mathfrak{K}$ and $\eta=1_{\mathfrak{K}}: \mathfrak{K} \longrightarrow \mathfrak{K}$. Moreover there exists always a maximal $\mathfrak{M} \subset \overline{\mathfrak{M}}$ such that $\mathfrak{K} / \mathfrak{M} \approx \mathfrak{K} / \overline{\mathfrak{M}}$. In the previous example $\mathfrak{M}_{2}$ is maximal.

Applications of this construction abound:

1) Let $\mathfrak{K}$ be a suitable category of topological spaces with or without base-points and let $\mathfrak{M}$ be the class of all inclusions $i_{k}: X \subset X \times I, i_{k}(x)=(x, k), k=0,1$, then $\mathfrak{K} / \mathfrak{M}$ is the homotopy category of $\mathfrak{K}$. The maximal $\overline{\mathfrak{M}}$ is therefore the class of all homotopy equivalences. More generally the authors of [17] establish their "derived category" $\mathfrak{D}_{S}$ by inverting all weak equivalences in the category $\mathfrak{M}_{S}$ of S-module spectra. Incidentally $\mathfrak{D}_{S}$ reveals itself to be equivalent to the Boardman category (cf. example 3 below).

2) Let $\mathfrak{K} \stackrel{\Phi}{\rightarrow} \mathfrak{L} \stackrel{\Psi}{\rightarrow} \mathfrak{K}$ be a pair of adjoint functors, appearing together with specific natural transformations $\alpha: \Phi \Psi \longrightarrow 1_{\mathfrak{L}}, \beta: 1_{\mathfrak{K}} \longrightarrow \Psi \Phi$. It turns out that $\mathfrak{K} /\{\beta\} \approx \mathfrak{L} /\{\alpha\}$. Many equivalences of categories are of this kind:

a) The homotopy category of $\mathrm{CW}$ spaces is equivalent to the homotopy category of Kancomplexes.

b) The Boardman-homotopy-category of spectra is equivalent to the homotopy category of simplicial Kan-spectra [9], [23].

3) The Boardman category of spectra itself is a quotient category of the category of spectra and functions of spectra as morphisms with $\mathfrak{M}$ being the class of all so-called cofinal embeddings of spectra:

A (CW) spectrum $E=\left\{E_{n}, n \in \mathbb{Z}\right\}$ is a sequence of based (CW) spaces coming together with mappings $\sigma: \Sigma E_{n} \longrightarrow E_{n+1}$. A function of spectra $f: E \longrightarrow F$ is simply a sequence $f_{n}: E_{n} \longrightarrow F_{n}, n \in \mathbb{Z}$, of continuous mappings, compatible with the bonding maps $\sigma$.

A function $m: E \subset F$ between CW spectra (i.e. $m_{n}: E_{n} \subset F_{n}$ ) is called cofinal whenever for any finite CW complex $K \subset F_{n}$ there is an index $k$ such that $K^{\prime} \subset F_{n+k}$ lies in the image of $m_{n+k}$, where $K^{\prime}$ denotes the image of $\Sigma^{k} K$ under

$$
\Sigma^{k} F_{n} \stackrel{\Sigma^{k-1} \sigma}{\longrightarrow} \Sigma^{k-1} F_{n+1} \longrightarrow \cdots \longrightarrow F_{n+k} .
$$

This can also be formulated for spectra where $E_{n}$ is only a based space (not a CW space) and $K$ a compact subspace of $F_{n}$.

4) Let $\mathfrak{K}$ be the category of based compacta (i.e. of based compact metric spaces), $\mathfrak{M}$ the class of all SSDR-maps [16], then $\mathfrak{K} / \mathfrak{M}$ is the strong shape category (of compact metric spaces). Incidentally ordinary shape does not admit a representation as a quotient category of $\mathfrak{K}$.

5 ) Let $M$ be a set of non-zero-divisors in some unital ring $R$, then one can localize $R$ at $M$. There are many different ways to express this algebraically. This fits into our scheme by converting $R$ into a category with one object and morphisms in 1-1 correspondence with the elements of $R$. If $R=\mathbb{Z}, M=\mathbb{Z} \backslash\{0\}$, we obtain the rationals $\mathbb{Q}$.

6) Let $\Phi: \mathfrak{K} \longrightarrow \mathbf{A b}$ (or $\mathbf{A} \mathbf{b}^{\mathbb{Z}}$ ) be any functor into the category of abelian groups or of $\mathbb{Z}$ graded abelian groups. Setting $\mathfrak{M}_{\Phi}=\{m \in \mathfrak{K} \mid \Phi(m)=$ isomorphism $\}$ establishes the category $\mathfrak{K} / \Phi=\mathfrak{K} / \mathfrak{M}_{\Phi}$.

We call an object $A \in \mathfrak{K} \Phi$-acyclic whenever $\Phi(A)=0$.

The class $\mathfrak{M}_{\Phi}$ is in this case maximal. 
1.1. Definition: An object $A \in \mathfrak{K}$ is $\mathfrak{M}$-local ( $\mathfrak{M}$-colocal) whenever for any $m \in \overline{\mathfrak{M}}, m$ : $X \longrightarrow Y$

$$
m^{*}: \mathfrak{K}(Y, A) \underset{\sim}{\approx} \mathfrak{K}(X, A)
$$

(resp.

$$
\left.m_{*}: \mathfrak{K}(A, X) \stackrel{\approx}{\rightarrow} \mathfrak{K}(A, Y)\right)
$$

is an isomorphism.

The following conditions require that there are sufficiently many $\mathfrak{M}$-(co-)local objects:

L) To any $A \in \mathfrak{K}$ there exist an $\mathfrak{M}$ - local object $A_{\mathfrak{M}}$ and $a\left(\eta: A \longrightarrow A_{\mathfrak{M}}\right) \in \overline{\mathfrak{M}}$ in a natural way.

$\left.\mathrm{L}^{*}\right) \quad$ To any $A \in \mathfrak{K}$ there exist an $\mathfrak{M}$-colocal object $A^{\mathfrak{M}}$ and $a\left(\eta: A^{\mathfrak{M}} \longrightarrow A\right) \in \overline{\mathfrak{M}}$ in a natural way.

At this stage of affairs we do not discuss the question how realistic such an assumption is.

Returning to example 6) suppose $\mathfrak{K}_{h}^{2}$ is a suitable homotopy category of pairs of topological spaces and $\Phi=h_{*}$ a generalized homology theory (cf. $\S 2$ ), then a simple exactness argument implies:

An object $A \in \mathfrak{K}$ is $\Phi$-(co-)local, if and only if

$$
\mathfrak{K}_{h}(X, A)=0 \quad\left(\mathfrak{K}_{h}(A, X)=0\right)
$$

for $\Phi$-acyclic $X$.

We have the following result:

1.2. Theorem: Suppose $L)\left(L^{*}\right)$ ) is satisfied, then $\mathfrak{K} / \mathfrak{M}$ and the full subcategory $\mathfrak{K}_{\mathfrak{M}}\left(\mathfrak{K}^{\mathfrak{M}}\right)$ $\subset \mathfrak{K}$ of all $\mathfrak{M}$-local (M-colocal) objects are equivalent.

Proof: It suffices to prove the colocal case, the other one is dual.

1.3. Lemma: Let $a \in \mathfrak{K}^{\mathfrak{M}}(X, Y)$, then

$$
a=\text { isomorphism } \Longleftrightarrow a \in \overline{\mathfrak{M}} \text {. }
$$

Proof: $\Longrightarrow$ : is trivial. $\Longleftarrow$ : Since $Y$ is $\mathfrak{M}$-colocal,

$$
a_{*}: \mathfrak{K}(Y, X) \stackrel{\approx}{\rightarrow} \mathfrak{K}(Y, Y)
$$

is an isomorphism. Hence we detect $\bar{a}: Y \longrightarrow X$ such that $a \bar{a}=1_{Y}$. On the other hand we deduce

$$
a(\bar{a} a)=(a \bar{a}) a=a 1_{X}
$$

implying $\bar{a} a=1_{X} \cdot-$

The assignment $A \longmapsto A^{\mathfrak{M}}$ establishes a functor ()$^{\mathfrak{M}}: \mathfrak{K} \longrightarrow \mathfrak{K}^{\mathfrak{M}}$ on the objects. Let $f \in \mathfrak{K}(A, B)$ be a morphism, then $B^{\mathfrak{M}} \mathfrak{M}$-colocal and $\left(\eta: B^{\mathfrak{M}} \longrightarrow B\right) \in \overline{\mathfrak{M}}$ imply the existence of a unique $f^{\mathfrak{M}}$ fitting into the diagram 


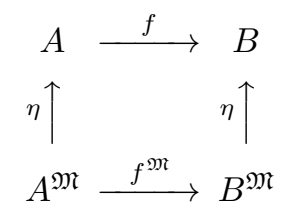

The functoriality of ( $)^{\mathfrak{M}}$ is now standard.

There is an inclusion $i^{\prime}: \mathfrak{K}^{\mathfrak{M}} \subset \mathfrak{K}$ and a functor $i=\beta i^{\prime}: \mathfrak{K}^{\mathfrak{M}} \longrightarrow \mathfrak{K} / \mathfrak{M}$. Lemma 1.3. ensures that $m^{\mathfrak{M}}$ is an isomorphism for $m \in \overline{\mathfrak{M}}$. So there exists a unique $\bar{\rho}: \mathfrak{K} / \mathfrak{M} \longrightarrow \mathfrak{K}^{\mathfrak{M}}$ such that $\bar{\rho} \beta=\rho=()^{\mathfrak{M}}$.

The isomorphisms

$$
\begin{gathered}
\bar{\rho} i \stackrel{\approx}{\stackrel{\sim}{\rightarrow}} 1_{\mathfrak{K} \mathfrak{M}} \\
1_{\mathfrak{K} / \mathfrak{M}} \stackrel{\approx}{\rightarrow} i \bar{\rho}
\end{gathered}
$$

are easily deduced from the existence of $\eta: A \longrightarrow A^{\mathfrak{M}}$.

Remarks: 1) Generally there is not much hope to have L), $L^{*}$ ) satisfied. In the previous example 4) there is a $\mathfrak{M}$-local $A \longmapsto|A|$ (terminology of [16]) but not in the category of compacta $(|A|$ is only metrizable, but, even for compact $A$, not compact).

In example 1) we cannot expect to find for any space $X$ a homotopy equivalent $\bar{X}$ such that

$$
f: X \simeq Y \Longleftrightarrow \bar{f}: \bar{X} \approx \bar{Y} .
$$

3) Dealing with example 6) we will henceforth talk about $\Phi$-(co-)local instead of $\mathfrak{M}_{\Phi}$-(co-)local objects. 


\section{Stable Homotopy Categories}

The world of homotopy theory is the homotopy category $\mathfrak{K}_{h}$ (cf. example 1$)$ in $\S 1$ ). It turns out that for based spaces $X=\left(X, x_{0}\right), Y=\left(Y, y_{0}\right) \quad[\Sigma X, Y]_{0}=\mathfrak{K}_{h}\left(\Sigma\left(X, x_{0}\right),\left(Y, y_{0}\right)\right)(\Sigma$ $=$ reduced suspension) carries a natural group structure, while $\left[\Sigma^{2} X, Y\right]_{0}$ carries an abelian group structure.

Thus homotopy theory is a bridge from point-set to algebraic topology.

Generally suspensions cannot be inverted. However there are homotopy invariants which commute with suspensions (e.g. for a homology theory $h_{*}$ on $\mathfrak{K}$ one has $h_{n}(X) \approx h_{n+1}(\Sigma X)$ ). These invariants are stable homotopy invariants. Stable homotopy theory lives in a stable homotopy category. An appropriate model for such a category is still the Boardman category of CW spectra (example 3 ) in $\S 1$ ). Since there is no way to desuspend every given space $X$, the objects of a stable homotopy category can never be topological spaces but something more fancy (like spectra).

Every spectrum $E$ (i.e. every object in the Boardman category) determines a homology theory on $\mathfrak{K}$ :

$$
E_{n}\left(X, x_{0}\right)=\left\{S^{n}, X \wedge E\right\}=\underset{k}{\underset{\lim }{\longrightarrow}}\left[S^{n+k}, X \wedge E_{k}\right]_{0} .
$$

It turns out that any (generalized) homology theory $h_{*}$ (i.e. any homology theory satisfying the Eilenberg-Steenrod axioms without the dimension axiom) is of this kind, whenever $h_{*}$ is defined for CW spaces and has compact carriers. The latter means that everything happens already on a compact subspace of $X$

$$
h_{n}(X)=\underset{K}{\lim } h_{n}(K), \quad K \subset X \text { compact. }
$$

If one investigates homology theories without that property then

$$
h_{n}()=\{E,\}_{n},
$$

'E' a spectrum, is a good candidate because (2) does not have compact carriers unless $E$ is a compact spectrum. The homology theories (1) and (2) can be extended for variables $X$ resp. ( ) which are not spaces, but spectra themselves. For this purpose one needs a $\wedge$-product in the Boardman category, which is associative up to homotopy.

More recent work of A. D. Elmendorf, I. Kriz, M. A. Mandell and J. P. May [17] established a $\wedge$-product in a stable homotopy category which is strictly associative and commutative (not only up to homotopy) and therefore very suitable for translating algebraic machineries into stable homotopy theory. Moreover this category turns out to be on the homotopy level equivalent to the Boardman category.

There is some work in progress about symmetric spectra [22], serving the same purpose.

An axiomatic treatment of stable homotopy theory is the subject of [21].

Let $E, A$ be spectra, then A. K. Bousfield [12] discovered an $E$ - localization (in our terminology an $E_{*}$-localization) consisting of a natural short exact sequence (this makes sense in the Boardman category with its rich algebraic structure)

$$
{ }_{E} A \longrightarrow A \stackrel{\eta}{\longrightarrow} A_{E}
$$

where ${ }_{E} A$ is $E$-acyclic, $A_{E} \quad E$-local and $\eta$ an $E$-isomorphism (= $E_{*}$-isomorphism).

There is no corresponding $E$-colocalization sequence for a reason which will become appearent in $\S 6$.

Using the terminology of $\S 1$, we can observe that in this case $\mathrm{L}$ ) holds but not $\mathrm{L}^{*}$ ). 


\section{Chain Functors}

Let $\mathfrak{K}$ be a category of pairs of topological spaces and $h_{*}()=\left\{h_{n} ; n \in \mathbb{Z}\right\}$ a generalized homology theory on $\mathfrak{K}$.

We are looking for a functor $C_{*}: \mathfrak{K} \longrightarrow \boldsymbol{c h}$ (= category of free chain complexes with natural bases), such that

1) $H_{*}\left(C_{*}(X, A)\right) \approx h_{*}(X, A)$

2) to each pair $(X, A) \in \mathfrak{K}$ there exists a short exact sequence

$$
0 \longrightarrow C_{*}(A) \stackrel{i_{\#}}{\longrightarrow} C_{*}(X) \stackrel{j_{\#}}{\longrightarrow} C_{*}(X, A) \longrightarrow 0
$$

( $i: A \subset X, j: X \subset(X, A))$ which determines the boundary

$$
\partial: h_{n}(X, A) \longrightarrow h_{n-1}(A) .
$$

A homology theory associated with such a functor $C_{*}$ is called flat. Ordinary singular homology is clearly flat: $C_{*}(X)$ is the singular chain complex of a space $X$, while $C_{*}(X, A)=$ $C_{*}(X) / i m i_{\#}$.

It turns out (R. O. Burdick, P. E. Conner and E. E. Floyd, [15]) that for $\mathfrak{K}=$ category of CW pairs such a natural chain complex does not exists unless $h_{*}$ is a direct sum of ordinary homology theories (i.e. of those which satisfy a dimension axiom).

On the other hand chain complexes are very suitable and convenient for all algebraic applications. It is much easier to work with individual cycles, boundaries etc, than with homology classes. A classical Künneth formula for example is firstly verified for tensor products of chain complexes. It does not hold for arbitrary generalized homology theories.

Therefore it is reasonable to look for an appropriate substitute for such a functor $C_{*}$ which works for arbitrary generalized homology theories.

At this point chain functors appear [2], [8]:

Let $C_{*}: \mathfrak{K} \longrightarrow \boldsymbol{c h}, C_{*}^{\prime}: \mathfrak{K} \longrightarrow \boldsymbol{c h}$ be functors such that $l: C_{*}^{\prime} \subset C_{*}$ is a subfunctor, assume furthermore that $i^{\prime}: C_{*}(A) \subset C_{*}^{\prime}(X, A)$ is a natural inclusion and that there exist non-natural chain mappings $\kappa: C_{*}(X) \longrightarrow C_{*}^{\prime}(X, A), \quad \varphi: C_{*}^{\prime}(X, A) \longrightarrow C_{*}(X)$ and display the diagram:

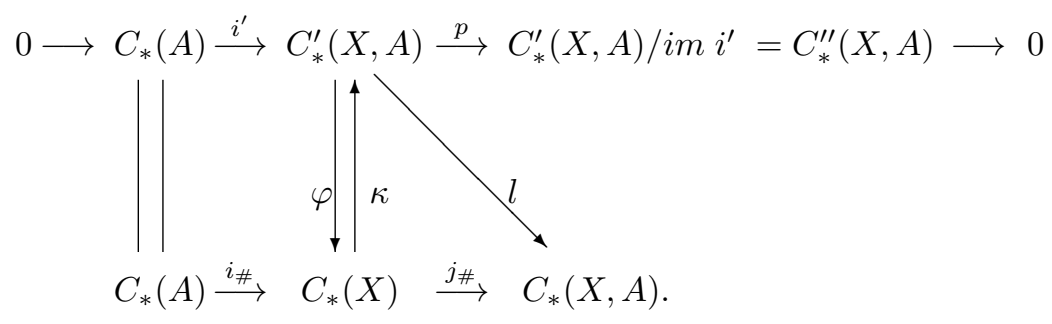

We require:

1) There exist (of course non-natural) chain homotopies resp. identities:

$$
\varphi \kappa \simeq 1, \quad j_{\#} \varphi \simeq l, \quad \kappa i_{\#}=i^{\prime} .
$$

2) $C_{*}(X, X)$ is acyclic; $C_{*}(\emptyset, \emptyset)=0$.

3) To any $z \in Z_{n}\left(C_{*}(X, A)\right)$ there exists a $z^{\prime} \in C_{*}^{\prime}(X, A), \bar{a} \in C_{n}(A, A)$ such that

$$
z \sim l z^{\prime}+s_{\#}(\bar{a}), \quad s:(A, A) \subset(X, A)
$$


$d z^{\prime}=-i^{\prime}(a)$, for $a \in C_{*}(A), q_{\#}(a)=d \bar{a}, q: A \subset(A, A)$.

This implies that $z^{\prime}$ is not a cycle but a chain with boundary in $i m i^{\prime}$. If $d z^{\prime} \in i m i^{\prime}$, there must be according to 2) a $\bar{a} \in C_{n}(A, A)$ such that $q_{\#}\left(i^{\prime}\right)^{-1} d z^{\prime}=d \bar{a}$.

Property 3$)$ implies that the homomorphism

$$
\begin{aligned}
& \psi: H_{*}\left(C_{*}^{\prime \prime}(X, A)\right) \longrightarrow H_{*}\left(C_{*}(X, A)\right) \\
& {\left[z^{\prime}\right] \quad \longmapsto \quad\left[l\left(z^{\prime}\right)+s_{\#}(\bar{a})\right]}
\end{aligned}
$$

is an epimorphism.

Since the top row of (2) is exact, there exists a $\bar{\partial}: H_{n}\left(C_{*}^{\prime \prime}(X, A)\right) \longrightarrow H_{n-1}\left(C_{*}(A)\right)$ and we require 4) $\operatorname{ker} \bar{\partial} \supset \operatorname{ker} \psi$

5) $\operatorname{ker} j_{*} \subset \operatorname{ker} p_{*} \kappa_{*}$

with $\kappa_{*}: H_{*}\left(C_{*}(X)\right) \longrightarrow H_{*}\left(C_{*}^{\prime}(X, A)\right)$, the mapping induced by $\kappa$.

6) a) Inclusions $i:(X, A) \subset(Y, B)$ induce monomorphisms onto a direct summand of $C_{*}(Y, B)$; im $i^{\prime}$, im l are direct summands of $C_{*}^{\prime}(X, A)$ resp. of $C_{*}(X, A)$.

b) If $i:(X \backslash U, A \backslash U) \subset(X, A)$ is an excision map (i.e. $\bar{U} \subset$ Int $\left._{X} A\right)$, then

$$
i_{*}: H_{*}\left(C_{*}(X \backslash U, A \backslash U)\right) \stackrel{\approx}{\rightarrow} H_{*}\left(C_{*}(X, A)\right)
$$

is an isomorphism.

c) Let $D:(X, A) \times I \longrightarrow(Y, B)$ be a homotopy, then there exists a natural, with $i^{\prime}$ and $l$ compatible chain homotopy $D_{\#}: C_{*+1}(X, A) \longrightarrow C_{*}(Y, B)$.

3.1. Definition: If all this structure is given, we call $\boldsymbol{C}_{*}=\left\{C_{*}, C_{*}^{\prime}, l, i^{\prime}, \varphi, \kappa\right\}$ a chain functor.

The derived homology theory of $\boldsymbol{C}_{*}$ is defined by

$$
h_{*}(X, A)=H_{*}\left(C_{*}(X, A)\right)
$$

on the homology groups, correspondingly for the induced mappings.

Let $[z] \in h_{*}(X, A)=H_{n}\left(C_{*}(X, A)\right)$ be given, then we detect according to axiom 3$)$ a $z^{\prime} \in C_{n}^{\prime}(X, A)$ with $d z^{\prime} \in i m i^{\prime}$ and $\psi\left[p\left(z^{\prime}\right)\right]=[z]$ and set

$$
\partial[z]=\left[\left(i^{\prime}\right)^{-1} d z^{\prime}\right]
$$

Axiom 4) ensures that $\partial[z]$ depends only on the class $[z]$ and not upon the choice of $z^{\prime}$. We get:

3.2. Theorem: 1) $h_{*}=\left\{h_{n} ; \partial\right\}$ is a generalized homology theory. Compact carriers for $\boldsymbol{C}_{*}$ imply compact carrier for $h_{*}$.

2) Any homology theory $h_{*}$ is isomorphic to the derived homology theory of a chain functor.

The first part follows from [8] proposition A2; the second part follows from [8] theorem 8.1.The concept of a chain functor is the appropriate substitute of the natural chain complexes $C_{*}$ at the beginning of this section which do not exist in general.

The elements $z^{\prime} \in C_{*}^{\prime}(X, A) \subset C_{*}(X, A)$ with $d z^{\prime} \in i m i^{\prime}$ are, what was historically called relative cycles (i.e. chains in $C_{*}(X)$ such that $d z^{\prime} \in C_{*-1}(A)$ ), cf. $\S 8$ for an example.

A flat homology theory $h_{*}$, is one which is associated with a functor $C_{*}: \mathfrak{K} \longrightarrow \boldsymbol{c h}$ satisfying (1). A chain functor $\boldsymbol{C}_{*}=\left\{C_{*}, C_{*}^{\prime}, l, i^{\prime}, \varphi, \kappa\right\}$ is called flat whenever $\varphi, \kappa$, and the associated homotopies $\varphi \kappa \simeq 1, j_{\#} \varphi \simeq l$ are natural.

We have:

3.3. Theorem: A homology theory is flat, if and only if there exists an associated flat chain functor.

One direction of this assertion is new. Therefore and because it furnishes a good example of a 
chain functor, we decided to include a proof of this theorem in $\S 8$.

3.4. Corollary: For a homology theory $h_{*}$ which is defined on the category of $C W$-spaces the following conditions are equivalent:

a) $h_{*}$ is the direct sum of ordinary homology theories;

b) $h_{*}$ is flat;

c) there exists a flat chain functor associated with $h_{*}$.

Follows immediately from 3.3. and a result, mentioned at the beginning of this section.

Remarks: 1) There are examples of homology theories $h_{*}$ which are "very close" to an ordinary homology theory because there exists an associated chain functor, which "almost" satisfies (1). Any kind of bordism theory $\Omega_{*}($ ) is of that form: The free abelian groups generated by singular manifolds, which ultimately determine the bordism-homology, behave like singular chain groups.

However the chain groups $C_{*}(X, A)$ of an associated chain functor have apart from singular manifolds (which form the the so-called models) more generators. Here it is possible to find specific chain functors with additional pleasant properties (chain functors with models, cf. [6]). 2) There have been many different attempts in the past, to "geometrize" generalized homology theories $h_{*}$ ( ). (cf. [14], [25], [26]). Most of them deal with bordism theories or try to endow $h_{*}$ with the structure of some kind of generalized bordism theory [14]. Some others try to replace differential graded abelian chain groups by differential abelian monoids [25], differential abelian loops [26] or differential graded groups [1]. The first attempt into this direction goes back to [19].

There are many relations between these different approaches and the present theory of chain functors; however, they cannot be viewed as predecessors of our theory. One, but not the only reason is, that this theory is not restricted to "good" spaces (CW- spaces) cf. $§ 7$.

3) There are no simple examples for chain functors for non-ordinary homology theories, which can be displayed in a few lines. Even the case of $h_{*}()=\pi_{*}(\boldsymbol{E} \wedge), \boldsymbol{E}$ a spectrum, requires some extensive technical preparation. One of the main problems is embodied in the fact that a group structure up to homotopy "o", establishing the correct homology groups, has to be replaced by a strict abelian group structure " +". Incidentally, this is a problem which also appears in other parts of stable homotopy theory (cf. [17]).

Let $\boldsymbol{C}_{*}$ be a chain functor with compact carriers on the category of CW pairs.

3.5. Theorem: [7] There exists a $C W$ spectrum (or, equivalently: a Kan spectrum cf.[23]) $\left|\boldsymbol{C}_{*}\right|$ such that

$$
\left|\boldsymbol{C}_{*}\right|_{*}() \approx H_{*}\left(\boldsymbol{C}_{*}()\right)
$$

That means that algebraic objects like chain functors can be realized as something so geometrical like a $\mathrm{CW}$ spectrum and vice-versa: A spectrum $E$ determines a homology theory $E_{*}($ ) which stems from a chain functor $\boldsymbol{C}(E)_{*}($ ) whose realization yields a spectrum such that the associated homology theory $\left|\boldsymbol{C}(E)_{*}\right|_{*}$ is isomorphic to $E_{*}()$.

Hence $E$ and $\left|\boldsymbol{C}(E)_{*}\right|$ are isomorphic as spectra in the Boardman-homotopy category.

Therefore the homotopy category of chain functors appears as a good candidate for a stable homotopy category (in fact modulo so-called phantom maps cf. [24] ch.5.3).

To this end we must define mappings (transformations) between chain functors:

Let $\lambda: K_{*} \longrightarrow L_{*}$ a natural transformation, then $\lambda$ is called a transformation of chain functors whenever it commutes with $l, i^{\prime}$, and the natural chain homotopies in 6c), (but not necessarily with $\varphi$ and $\kappa$ ). 
This establishes the category of chain functors $\mathfrak{C} h$.

We can introduce homotopies between such transformations, accomplishing homotopy classes of transformations, $\left[\boldsymbol{K}_{*}, \boldsymbol{L}_{*}\right]$.

As a result we have the homotopy category of chain functors $\mathfrak{C} h_{h}$.

One could of course in addition require that a transformation of chain functors commutes with all structures of a chain functor, i.e. with $\kappa, \varphi$ and the chain homotopies $\varphi \kappa \simeq 1, j_{\#} \varphi \simeq l$.

Is this a very strong requirement?

A g-transformation $\lambda: \mathbf{K}_{*} \longrightarrow \mathbf{L}_{*}$ is one which satisfies the condition that $i m \lambda \subset L_{*}()$ is always a direct summand. By changing $\kappa, \varphi$, etc on $i m \lambda$ seperately we obtain:

3.6. Proposition: ([2] 4.4.) Every g-transformation $\lambda$ allows a factorization as $\lambda=\varepsilon \tilde{\lambda}$, where $\varepsilon$ is an isomorphism in $\mathfrak{C} h$ and $\tilde{\lambda}$ preserves all structure of a chain functor.

How far is a given $\lambda \in \mathfrak{C} h\left(\mathbf{K}_{*}, \mathbf{L}_{*}\right)$ from a g-transformation?

Here we have:

3.7. Proposition: ([2] 4.5.) To every $\lambda \in \mathfrak{C} h\left(\mathbf{K}_{*}, \mathbf{L}_{*}\right)$ there exists a g-transformation $\tilde{\lambda}$ and a chain homotopy equivalence $\gamma$ such that

$$
\tilde{\lambda}=\gamma \lambda
$$

Thus, every $\lambda$ satisfies the stronger compatibility requirements up to a homotopy equivalence. At this stage it is worth asking what happens if certain properties of a chain functor are waived.

Without $6 \mathrm{~b})$ and the requirement of compact carriers there is no geometric realization $\left|\boldsymbol{C}_{*}\right|$. This observation will become crucial in $\S 6$.

If we relinquish the requirement that $i^{\prime}$ is an inclusion, then we still have homology groups but not the top exact sequence in (2) and therefore no boundary operator.

The stronger versions, requiring that $i m i^{\prime}$ etc are direct summands is satisfied for the chain functor, which was explicitely constructed in [8] and needed in [3].

Condition $6 \mathrm{~b}$ ) guarantees that the associated homology is in fact a homology theory, not only a pre-homology theory (cf. [8]).

Nevertheless there is good reason to consider also so-called irregular chain functors which do not satisfy 6 )a), b) and where $i^{\prime}, l$ and mappings induced by inclusions are not necessarily monic. In particular $C_{*}^{\prime}$ is not necessarily a subfunctor of $C_{*}$.

The concept of a transformation between (irregular) chain functors can be established.

As an example of such an irregular chain functor we introduced in [3] $\S 3$ the (irregular) chain functor $\mathbb{Z}_{*}$ :

$$
\mathbb{Z}_{n}^{\prime}(X, A)=\mathbb{Z}_{n}(X)=\left\{\begin{array}{l}
<z_{X}>\mid d z_{X}=0 \cdots n=0, X \neq \emptyset \\
0 \quad \cdots \text { elsewhere }
\end{array}\right.
$$

$(<\cdots\rangle=$ free abelian group, generated by $\ldots)$,

$$
\mathbb{Z}_{n}(X, A)=0 \cdots A \neq \emptyset .
$$

The morphisms $l, i^{\prime} \varphi \kappa$ are immediately established, furnishing the structure of an (irregular) chain functor.

The derived homology is not a homology theory, because 


$$
\begin{aligned}
& H_{n}\left(\mathbb{Z}_{*}\right)(X)=\left\{\begin{array}{l}
\mathbb{Z} \cdots X \neq \emptyset, n=0 \\
0 \cdots \text { elsewhere }
\end{array}\right. \\
& H_{n}\left(\mathbb{Z}_{*}\right)(X, A)=0 \cdots A \neq \emptyset, n \in \mathbb{Z},
\end{aligned}
$$

which can never happen within a homology theory.

Irregular chain functors and chain functors without compact carriers (which cannot be realized as spectra) are necessary in order to be able to perform certain operation between chain functors, which lead outside of the category of spectra but which are still available for (eventually irregular) chain functors.

We will encounter several examples in the next sections. 


\section{Tensor Products of Spectra}

Let $\boldsymbol{K}_{*}, \boldsymbol{L}_{*}$ be two regular chain functors, then one can define a tensor product $\boldsymbol{K}_{*} \otimes \boldsymbol{L}_{*}$ :

$$
\begin{gathered}
(K \otimes L)_{n}(X, A)=\bigoplus_{p+q=n} K_{p}(X, A) \otimes L_{q}(X, A) \\
(K \otimes L)_{n}^{\prime}(X, A)=\bigoplus_{p+q=n} K_{p}^{\prime}(X, A) \otimes L_{q}^{\prime}(X, A) \oplus \bigoplus_{p+q=n} K_{p}^{\prime}(X, A) \otimes L_{q}(A, A) \oplus \\
\oplus \bigoplus_{p+q=n} K_{p}(A, A) \otimes L_{q}^{\prime}(X, A) .
\end{gathered}
$$

This can be endowed with the additional structure of a chain functor [3].

There is a tensor product for irregular chain functors, which has to be defined a little differently.

4.1. Theorem: The tensor product of two (regular) chain functors is a (regular) chain functor.

It turns out that for any chain functor $\boldsymbol{K}_{*}$ (regular or irregular) there exists an isomorphism of irregular chain functors

$$
(K \otimes \mathbb{Z})_{*} \approx K_{*} .
$$

So $\mathbb{Z}_{*}$ operates like an identity. Notice that (even for regular $\left.\boldsymbol{K}_{*}\right)(\boldsymbol{K} \otimes \mathbb{Z})_{*}$ is not regular.

The classical proof of a Künneth formula (for chain complexes) carries immediately over to chain functors, yielding

4.2. Theorem: Let $\boldsymbol{K}_{*}, \boldsymbol{L}_{*}$ be regular chain functors, then there exists a, in $\boldsymbol{K}_{*} \boldsymbol{L}_{*}$ as well as in $(X, A)$, natural short exact sequence

$$
\begin{aligned}
0 \longrightarrow\left(H_{*}\left(\boldsymbol{K}_{*}\right)(X, A)\right. & \left.\otimes H_{*}\left(\boldsymbol{L}_{*}\right)(X, A)\right)_{n} \longrightarrow H_{n}(\boldsymbol{K} \otimes \boldsymbol{L})(X, A) \longrightarrow \\
& \longrightarrow \operatorname{Tor}\left(H_{*}\left(\boldsymbol{K}_{*}\right)(X, A), H_{*}\left(\boldsymbol{L}_{*}\right)(X, A)\right)_{n-1} \longrightarrow 0 .
\end{aligned}
$$

We have in the title of this section promised a tensor product between spectra rather than between chain functors. In order to be able to accomplish that, we need one more result about chain functors:

Let $\boldsymbol{K}_{*}, \boldsymbol{L}_{*}$ be regular chain functors with isomorphic homology, i.e. we assume the existence of a natural isomorphism $\gamma: H_{*}\left(\boldsymbol{K}_{*}\right)() \approx H_{*}\left(\boldsymbol{L}_{*}\right)\left(\right.$ ). What relationship exists between $\boldsymbol{K}_{*}$ and $\boldsymbol{L}_{*}$ ? Since every transformation between chain functors induces a transformation of the associated homology theories, one could ask whether $\gamma$ is induced by a natural transformation $\lambda: \boldsymbol{K}_{*} \longrightarrow \boldsymbol{L}_{*}$. This is not true, however we have a result, which comes close to it ([2] theorem 1.1.):

4.3. Theorem: Let $\gamma: H_{*}\left(\boldsymbol{K}_{*}\right)() \approx H_{*}\left(\boldsymbol{L}_{*}\right)(\mathrm{)}$ be a natural eqivalence of homology theories, then there exists a third chain functor $\boldsymbol{C}_{*}$ and transformations of chain functors

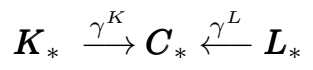

inducing isomorphisms of homology theories such that

$$
\gamma=\left(\gamma_{*}^{L}\right)^{-1} \gamma_{*}^{K}
$$


Let $E, F$ be spectra, then we find chain functors $\boldsymbol{C}(E)_{*}, \boldsymbol{C}(F)_{*}$ such that $\left.H_{*}\left(\boldsymbol{C}(E)_{*}\right)\right)(\mathrm{)}) \approx$ $E_{*}($ ) resp. for $F$. According to 3.1. these chain functors functorially. No we define:

$$
E \otimes F=\mid(\boldsymbol{C}(E) \otimes \boldsymbol{C}(F) \mid .
$$

According to theorem 4.3. the right-hand side is (up to an isomorphism in the Boardman homotopy category) well-defined. As a result, (5) establishes a tensor product of spectra up to the same kind of isomorphism.

The tensor product and the $\wedge$-product of spectra differ in a fundamental way. This is already exhibited by the existence of a Künneth formula in Theorem 4.2.. If one is looking for a functor, converting tensor products into $\wedge$-products or the other way round, then it appears to be unlikely that such a functor exists simply on the level of spectra, because units (the irregular and therefore not realizable chain functor $\boldsymbol{Z}_{*}$ for tensor products and the sphere spectrum for the $\wedge$ - product) would be expected to correspond under these functors.

There is an interesting application of tensor products for spectra:

As a corollary of theorem 4.2. we have a Künneth formula for tensor products for spectra rather than for chain functors:

$$
\begin{aligned}
0 \longrightarrow\left(E_{*}(X, A) \otimes\right. & \left.F_{*}(X, A)\right)_{n} \longrightarrow(E \otimes F)_{n}(X, A) \longrightarrow \\
& \longrightarrow \operatorname{Tor}\left(E_{*}(X, A), F_{*}(X, A)\right)_{n-1} \longrightarrow 0
\end{aligned}
$$

Let $G_{i} \in \boldsymbol{A} b, i=1,2$ be abelian groups and $K\left(G_{i}\right)$ the associated Eilenberg-MacLane spectra.

4.4. Theorem: Suppose that $\operatorname{Tor}\left(G_{1}, G_{2}\right)=0$, then there exists an isomorphism of spectra in the Boardman homotopy category

$$
K\left(G_{1}\right) \otimes K\left(G_{2}\right) \approx K\left(G_{1} \otimes G_{2}\right) .
$$

Proof: Application of (6) yields an exact sequence

$$
\begin{array}{r}
0 \longrightarrow\left(K\left(G_{1}\right)_{*}() \otimes K\left(G_{2}\right)_{*}()\right)_{n} \longrightarrow\left(K\left(G_{1}\right) \otimes K\left(G_{2}\right)\right)_{n}() \longrightarrow \\
\longrightarrow \operatorname{Tor}\left(K\left(G_{1}\right)_{*}(), K\left(G_{2}\right)_{*}()\right)_{n-1} \longrightarrow 0 .
\end{array}
$$

By inserting a point, $\left(K\left(G_{1}\right) \otimes K\left(G_{2}\right)\right)_{*}$ reveals itself as an ordinary homology theory with coefficient group $G_{1} \otimes G_{2}$. Hence (7) follows.- 


\section{Localizations}

We have already agreed to call $E_{*}$-localizations ( $E$ a spectrum) simply $E$-localizations. Chain functors, defined on a category of pairs of topological spaces (e.g. CW pairs) can be extended over a category of spectra.

It turns out that $E$-localizations of chain functors always exist (cf. [3]). Moreover for further applications it makes sense to generalize this process, which we are going to describe below, by replacing a single object $E$ by a whole subcategory $\mathfrak{L} \subset \mathfrak{K}$.

The concept of an $\mathfrak{L}$-acyclic chain functor $\boldsymbol{K}_{*}$ as well as that of an $\mathfrak{L}$ - isomorphism $\eta: \boldsymbol{K}_{*} \longrightarrow$ $\boldsymbol{L}_{*}$ is easily defined in complete analogy to the corresponding concepts in $\S 1$. A chain functor $\boldsymbol{A}_{*}$ is $\mathfrak{L}$-local, whenever

$$
\left[\boldsymbol{K}_{*}, \boldsymbol{A}_{*}\right]=0
$$

for all $\mathfrak{L}$-acyclic chain functors $\boldsymbol{K}_{*}$, denoting by $[\cdots, \cdots]$ the chain homotopy classes of chain functor transformations.

We have ([3] 5.6. Corollary):

5.1. Theorem To each subcategory $\mathfrak{L} \subset \mathfrak{K}$ and any chain functor $\boldsymbol{A}_{*}$, defined on $\mathfrak{K}$, there exists a natural exact sequence

$$
{ }_{\mathfrak{L}} \boldsymbol{A}_{*} \stackrel{\zeta}{\longrightarrow} \boldsymbol{A}_{*} \stackrel{\eta}{\longrightarrow} \boldsymbol{A}_{\mathfrak{L} *}
$$

with $\mathfrak{L}$-acyclic ${ }_{\mathfrak{L}} \boldsymbol{A}_{*}, \mathfrak{L}$-isomorphism $\eta$ and $\mathfrak{L}$ - local $\boldsymbol{A}_{\mathfrak{L} *}$

Exactness is understood either by establishing in the category of chain functors a triangulation (which can be accomplished by standard methods) or simply by defining suspensions $(\Sigma \boldsymbol{A})_{*}$ for chain functors analogously as this is done for chain complexes.

Suppose $\mathfrak{K}=\mathfrak{B}$ is the Boardman category of pairs of spectra, $\mathfrak{L}$ reduces to a single object $E$ and $\boldsymbol{A}_{*}$ is the chain functor $\boldsymbol{C}(F)_{*}$ associated with a spectrum $F$, then we regain the Bousfield exact localization sequence [11]

$$
{ }_{E} F \longrightarrow F \longrightarrow F_{E}
$$

Theorem 5.1. asserts that in our present case condition L) of $\S 1$ is satisfied.

The proof of theorem 5.1. consists of several steps:

1) Establishing ${ }_{\mathfrak{L}} \boldsymbol{A}_{*}$ :

11) There exists on the subcategory $\mathfrak{L}$ an acyclic chain functor $U_{*}$ and a g-transformation $\boldsymbol{U}_{*} \stackrel{\alpha}{\rightarrow} \boldsymbol{A}_{*} \mid \mathfrak{L}$ which is universal with respect to that property: For any other $\boldsymbol{V}_{*} \stackrel{\lambda}{\rightarrow} \boldsymbol{A}_{*} \mid \mathfrak{L}$, $V_{*}$ being an acyclic chain functor on $\mathfrak{L}$, one has a factorization of $\lambda=\alpha \zeta$. Moreover one can assume that $\zeta$ is a $g$ - transformation.

This is not difficult.

12) Let $\boldsymbol{C}_{*}$ be any chain functor, $\alpha: \boldsymbol{K}_{*} \longrightarrow \boldsymbol{C}_{*} \mid \mathfrak{L}$ a g-transformation, then there exists an extension $\overline{\boldsymbol{K}}_{*}$ of $\boldsymbol{K}_{*}$ now defined as a chain functor on $\mathfrak{K}$, a $\bar{\alpha}: \overline{\boldsymbol{K}}_{*} \longrightarrow \boldsymbol{C}_{*}$, and a $\tau: \boldsymbol{K}_{*} \subset \overline{\boldsymbol{K}}_{*} \mid \mathfrak{L}$ which is a strong deformation retract, such that $(\bar{\alpha} \mid \mathfrak{L}) \tau=\alpha$.

The construction $\overline{()}$ is universal: Let $\boldsymbol{L}_{*} \stackrel{\beta}{\rightarrow} \boldsymbol{C}_{*}$ be a g-transformation in $\mathfrak{K}, \gamma: \boldsymbol{L}_{*} \mid \mathfrak{L} \longrightarrow \boldsymbol{K}_{*}$ a transformation, such that $(\beta \mid \mathfrak{L})=\alpha \gamma$, then there exists an, up to chain homotopy unique, $r: \boldsymbol{L}_{*} \longrightarrow \overline{\boldsymbol{K}}_{*}$ such that $\bar{\alpha} r=\beta, \quad r \mid \mathfrak{L}=\tau \gamma$.

13) Apply 12) to the situation 11) and set

$$
\bar{U}_{*}={ }_{\mathfrak{L}} A_{*}, \quad \zeta=\bar{\alpha}: \bar{U}_{*} \longrightarrow A_{*} .
$$


It is easy to see, that $\bar{U}_{*}$ is $\mathfrak{L}$ - acyclic.

2) Establishing $\boldsymbol{A}_{\mathfrak{L} *}$ :

We define

$$
A_{\mathfrak{L} *}=A_{*} \cup_{\bar{\alpha}} \text { cone } \bar{U}_{*},
$$

where the cone-construction for chain functors works in the same way as for chain complexes. The existence of a transformation $\eta: \boldsymbol{A}_{*} \longrightarrow \boldsymbol{A}_{\mathfrak{L}_{*}}$ is now immediate.

The fact that $\mathfrak{C} h_{h}\left(\boldsymbol{V}_{*}, \boldsymbol{A}_{\mathfrak{L} *}\right)=0$, for $\mathfrak{L}$-acyclic $\boldsymbol{V}_{*}$ follows, because one can easily show that every $f: \boldsymbol{V}_{*} \longrightarrow \boldsymbol{A}_{\mathfrak{L} *}$ factors up to homotopy over the (contractible) cone $\overline{\boldsymbol{U}}_{*}$.

The difficulties with this proof are concentrated in the verification of the structure of a chain functor for $\overline{\boldsymbol{K}}_{*}$, in particular providing the different mappings $\varphi, \kappa, \cdots$ and the necessary chain homotopies in 12). On the level of chain complexes (without taking care of the additional structure of a chain functor) the construction of $\overline{\boldsymbol{K}}_{*}$ is standard.

There is another free option in this construction which is more than accidental: One can perform all this for chain functors with compact carriers to the effect that ${ }_{\mathfrak{L}} \boldsymbol{A}_{*}$ and $\boldsymbol{A}_{\mathfrak{L} *}$ have compact carriers and one can alternatively omit this assumption.

We are able to construct the exact sequence (1) also for irregular chain functors $\boldsymbol{A}_{*}$ yielding irregular chain functors ${ }_{\mathfrak{L}} \boldsymbol{A}_{*}$ and $\boldsymbol{A}_{\mathfrak{L} *}$. In particular we have $\mathfrak{L}$-localizations for $\boldsymbol{A}_{*}=\mathbb{Z}_{*}$. This is important because we are able to describe any $\mathfrak{L}$-localization of a regular chain functor $\boldsymbol{A}_{*}$ by temsoring with the sequence (1) for $\mathbb{Z}_{*}$ :

5.2. Theorem: There exist suitable $\simeq$-equivalences such that the sequence (1) is given by

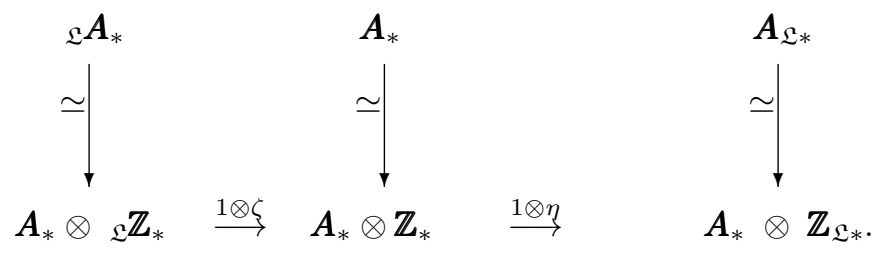

Remark: A very extensive study of (co-)localizations from a more general point of view is the subject of [20]. 


\section{Colocalizations}

As far as the definition is concerned colocalizations of chain functors [4] are entirely dual to localizations.

Again we have:

6.1. Theorem: Every chain functor $\boldsymbol{A}_{*}$ admits an exact colocalization sequence

$$
A_{*}^{\mathfrak{L}} \stackrel{\eta}{\longrightarrow} A_{*} \longrightarrow{ }^{\mathfrak{L}} A_{*}
$$

with $\mathfrak{L}$-acyclic ${ }^{\mathfrak{L}} \boldsymbol{A}_{*}, \mathfrak{L}$-colocal $\boldsymbol{A}_{*}^{\mathfrak{L}}$ and $\mathfrak{L}$-isomorphism $\eta$.

Thus the existence theorem 6.1. is dual to the existence theorem 5.1. for localizations. However the proofs are not dual.

We will again indicate the proof by omitting everything concerning the specific structure of a chain functor (i.e. the construction of $\varphi, \kappa, i^{\prime}, l$ etc) which in fact constitutes the real difficulty of the verification.

Unlike the situation in 5.1. we establish $\boldsymbol{A}_{*}^{\mathfrak{L}}$, the $\mathfrak{L}$-colocal chain functor, right-away:

Let $X \in \mathfrak{K}$ be any object, then we consider classes $(\zeta, f), \zeta \in A_{*}(L), f: L \longrightarrow X$ a mapping in $\mathfrak{K}$ (resp. everything for pairs $(X, A),\left(L, L^{6}\right)$, for the sake of simplicity we will confine ourselves to objects $X, L$ etc) and define a suitable quotient of the free abelian group generated by these pairs:

1)

$$
\left(\zeta_{1}, f\right)+\left(\zeta_{2}, f\right) \sim\left(\zeta_{1}+\zeta_{2}, f\right)
$$

2)

$$
(\zeta, f g) \sim\left(g_{\#} \zeta, f\right), \quad g \in \mathfrak{L}\left(L_{1}, L_{2}\right)
$$

whenever all pairs involved are defined. The group $A_{*}^{\mathfrak{L}}(X)$ is this quotient group. We have induced mappings

$$
h_{\#}(\zeta, f)=(\zeta, h f), \quad h \in \mathfrak{K}
$$

and a boundary

$$
d(\zeta, f)=(d \zeta, f)
$$

The transformation

$$
\begin{aligned}
\eta_{X}: A_{*}^{\mathfrak{L}}(X) & \longrightarrow A_{*}(X) \\
(\zeta, f) & \longmapsto f_{\#} \zeta
\end{aligned}
$$

is well-defined and an isomorphism on $\mathfrak{L}$.

Let $\boldsymbol{K}_{*}$ be any $\mathfrak{L}$-acyclic chain functor and $\alpha: \boldsymbol{A}_{*}^{\mathfrak{L}} \longrightarrow \boldsymbol{K}_{*}$ a transformation. We need the fact that every $\bar{\zeta} \in \boldsymbol{A}_{*}^{\mathfrak{L}}(X)$ comes from a $\zeta \in \boldsymbol{A}_{*}^{\mathfrak{L}}(L), L \in \mathfrak{L}$, i.e. that $\bar{\zeta}=f_{\#}(\zeta)$. Since a cycle $\alpha(\zeta) \in K_{*}(L)$ bounds $d \xi=\alpha(\zeta), \alpha(\bar{\zeta})=d\left(f_{\#} \xi\right)$ so that $\alpha(\bar{\zeta})$ is bounding. This implies that $\alpha \simeq 0$ and

$$
\left[\boldsymbol{A}_{*}^{\mathfrak{L}}, \boldsymbol{K}_{*}\right]=0 .
$$

The $\mathfrak{L}$-acyclic ${ }^{\mathfrak{L}} \boldsymbol{A}_{*}$ is defined by killing $\eta\left(\boldsymbol{A}_{*}\right)$ by means of a cone construction.-

Now suppose that $\mathfrak{L}$ reduces to a single object $E$, then it is obvious that in general $\boldsymbol{A}_{*}^{E}$ does not have compact carriers:

If $\zeta \in A_{*}(E), \quad f: E \longrightarrow X$, there will be no compact $i: K \subset X, \quad f^{\prime}: E \longrightarrow K, \quad \zeta^{\prime} \in$ $A_{*}(E)$ such that $(\zeta, f) \sim\left(\zeta^{\prime}, i f^{\prime}\right)$. 
Since $\boldsymbol{A}_{*}^{\mathfrak{L}}$ is easily seen to be unique with respect to the properties of theorem 6.1. , this does not depend on the construction. Going over to compact carriers (i.e. defining

$$
\left.\tilde{A}_{*}(X)=\underset{K}{\lim } A_{*}^{\mathfrak{L}}(K), \quad K \subset X \text { compact }\right)
$$

destroys immediately the $\mathfrak{L}$-isomorphism $\eta$, since it cannot be expected that to any $\zeta \in A_{*}(E)$ there exists a $\zeta^{\prime} \in A_{*}(E), \quad i: K \subset E, f: E \longrightarrow K$ such that $i_{\#} f_{\#} \zeta^{\prime}=\zeta$. So $A_{*}^{\mathfrak{L}}$ cannot be realized as a spectrum $\left|\boldsymbol{A}_{*}^{\mathfrak{L}}\right|$. Consequently there is no $E$-colocalization on the level of spectra, although the corresponding chain functor $\boldsymbol{A}_{*}^{\mathfrak{L}}$ exists.

However there is some remedy:

Let $E$ be a spectrum and consider the homology theory

$$
h_{*}()=\{E,\}_{*},
$$

which satisfies all properties of a homology theory, eventually without the existence of compact carriers. On the other hand there is a chain functor $\boldsymbol{A}_{*}$ associated with $h_{*}$ (of course again without compact carriers).

Now we profit from the flexibility of the constructions involved and invent new formal objects $D E, E \in \mathfrak{B}$ with mappings

$$
\begin{aligned}
& E_{1} \longrightarrow D E \quad \longleftrightarrow \quad E \wedge E_{1} \longrightarrow S^{0} \\
& D E_{1} \longrightarrow D E \quad \longleftrightarrow \quad E \longrightarrow E_{1} \\
& D E \longrightarrow E_{1} \longleftrightarrow S^{0} \longrightarrow E \wedge E_{1}
\end{aligned}
$$

where on the right-hand side one has mappings existing in $\mathfrak{B}$ and on the left side new mappings.

The homology theory $h_{*}$ can now be defined by

$$
h_{*}()=D E_{*}() .
$$

The construction of colocalization works also for this enlarged category $\overline{\mathfrak{B}} \supset \mathfrak{B}$, yielding $\boldsymbol{A}_{*}^{D E}$, also denoted by $\boldsymbol{A}_{*}^{[E,]}$.

It turns out that $\boldsymbol{A}_{*}^{D E}=\boldsymbol{A}_{*}^{[E,]}$ has compact carriers on $\mathfrak{B}$ :

Every $\bar{f}: D E \longrightarrow X$ in $\overline{\mathfrak{B}}$ is associated with a $f: S^{0} \longrightarrow E \wedge X$ in $\mathfrak{B}$, allowing a factorization over a compact $i: K \subset X \quad g: S^{0} \longrightarrow E \wedge K, \quad\left(1_{E} \wedge i\right) g=f$.

Hence one has $\bar{f}=i \bar{g}, \quad \bar{g}: D E \longrightarrow K$ being the new morphism, associated with $g$. So, unlike in the previous case, every $(\zeta, \bar{f})$ is of the form $i_{\#}(\zeta, \bar{g})$.

It should be observed that if $E$ is a finite spectrum, admitting an S-dual $D E$ (already in $\mathfrak{B}$ ), we can identify the new and the old $D E$.

As a result:

6.2. Theorem: To every spectrum $E \in \mathfrak{B}$ there exists an exact $[E$,$] -colocalization sequence$

$$
\boldsymbol{A}_{*}^{[E,]} \stackrel{\eta}{\longrightarrow} \boldsymbol{A}_{*} \longrightarrow{ }^{[E,]} \boldsymbol{A}_{*}
$$

with $[E$,$] -acyclic { }^{[E,]} \boldsymbol{A}_{*},[E$,$] -colocal \boldsymbol{A}_{*}^{[E,]}$ and $[E$,$] -isomorphism \eta$.

6.3. Corollary: Every spectrum $A \in \mathfrak{B}$ has an $[E$,$] -colocalization A^{[E,]}$.

This is simply $\left|\boldsymbol{A}_{*}^{[E,]}\right|, \quad \boldsymbol{A}_{*}=\boldsymbol{C}(A)_{*}$ the chain functor associated with $A$, i.e. $H_{*}(\boldsymbol{A})()=$ $A_{*}$ ( ) (cf. A.K. Bousfield [13]). 


\section{Strong Homology Theories}

The promised example of a $\mathfrak{L}$-localization process with respect to a category $\mathfrak{L} \subset \mathfrak{K}$ which does not consists just of a single object leads us to strong homology theories.

The history of strong homology theories goes back to the fourtieth of this century. On the category of compact metric pairs $\boldsymbol{C o m}^{2}$ lives a homology theory which exhibits many very pleasant properties:

1) It satisfies a strong excision axiom:

$$
h_{*}(X, A) \stackrel{p_{*}}{\approx} h_{*}(X / A, \star)
$$

is always an isomorphism.

2) It satisfies a cluster axiom:

If $\left(X, x_{0}\right)=\stackrel{C}{C=1}_{C}^{\infty}\left(X_{i}, x_{i 0}\right)$ is the cluster of spaces $\left(X_{i}, x_{i 0}\right)$, i.e. the wedge with the strong topology, then the inclusions $\left(X_{i}, x_{i 0}\right) \subset\left(X, x_{0}\right)$ induces an isomorphism

$$
h_{*}\left(X, x_{0}\right) \approx \prod_{i=1}^{\infty} h_{*}\left(X_{i}, x_{i 0}\right) .
$$

This kind of homology is called Steenrod-Sitnikov-homology. It turns out that $h_{*}$ is completely determined by its restriction to the category of compact CW pairs [10]. That means that for any spectrum $E \in \mathfrak{B}$ one is entitled to talk about the Steenrod-Sitnikov-homology theory $\bar{E}_{*}()$ which coincides with $E_{*}()$ on CW pairs. For ordinary homology theories this implies that $h_{*}$ is determined by its coefficient group $G$. This is a result of J. Milnor (cf. [10] for further references).

The cluster axiom (2) is a special case of a continuity property of a homology theory $h_{*}$, asserting that $h_{*}$ commutes up to isomorphisms with inverse limits. Since, according to a classical result of S. Eilenberg and N. Steenrod, no homology theory can satisfy such a continuity property in full generality, (2) is a reasonable substitute.

By looking for a set of axioms describing a good homology theory for more general spaces, one tries to detect some kind of continuity which is on one hand not equivalent to full continuity and which on the other hand is sufficiently strong to determine, together with the remaining Eilenberg- Steenrod-axioms for a generalized homology theory, including a suitable form of excison, a homology theory, which is now called strong homology theory.

This kind of continuity is continuity on the chain level (c-continuity): One defines the notion of a continuous chain functor and requires that every chain functor $\boldsymbol{C}_{*}$ which is associated with the strong homology theory $h_{*}$ under consideration can be transformed into a continuous chain functor (without changing the derived homology). Concerning the details the reader is referred to the literature mentioned in [5]. In particular one has again a uniqueness theorem asserting that a strong homology theory is completely determined by its values on "good" (i.e. $\mathrm{CW})$ spaces.

In the case of compact metric spaces this c-continuity turns out to be equivalent to the cluster axiom, so that Steenrod-Sitnikov-homology is a special case of strong homology.

Surprisingly there is the following relationship between strong homology theories and localizations:

7.1. Theorem: [5] Let $\mathfrak{K}$ be a category of pairs of topological spaces, $\mathfrak{L} \subset \mathfrak{K}$ the subcategory of $C W$ pairs, $\boldsymbol{A}_{*}$ a chain functor on $\mathfrak{K}$, then the derived homology of $\boldsymbol{A}_{\mathfrak{L} *}$ is the strong homology which coincides with $H_{*}\left(\boldsymbol{A}_{*} \mid \mathfrak{L}\right)($ ) on the subcategory $\mathfrak{L}$. 


\section{Proof of theorem 3.3.}

Suppose $h_{*}$ admits a functor $C_{*}: \mathfrak{K} \longrightarrow \boldsymbol{c h}$ satisfying $\S 3$ (1). By a standard argument we can assume that all inclusions induce monomorphisms into direct summands. We have $C_{*}(A, A)=0$ and set

$$
K_{*}(X, A)=C_{*}(X) \cup_{i} \text { cone } C_{*}(A)
$$

i.e. we attach the cone over $C_{*}(A)$ to $C_{*}(X)$ by means of the inclusion $i: A \subset X$. Then we define $K_{*}^{\prime}(X, A)=K_{*}(X)$ and assume that

$$
K_{*}^{\prime}(X, A) \stackrel{\varphi}{\longrightarrow} K_{*}(X) \stackrel{\kappa}{\longrightarrow} K_{*}^{\prime}(X, A)=K_{*}(X)
$$

are the identities.

A cycle $z=z^{\prime}+\bar{a}$ in $K_{*}(X, A), z^{\prime} \in C_{*}(X), \bar{a} \in$ cone $C_{*}(A)$ is a chain, satisfying $d z^{\prime}=-d \bar{a} \in$ $C_{*-1}(A)$. Such a $z$ bounds whenever there exists a $x=x^{\prime}+\bar{b}$ such that

$$
d x=d x^{\prime}+d \bar{b}=z^{\prime}+\bar{a}, \quad \bar{a} . \bar{b} \in \text { cone } C_{*}(A) .
$$

The homology class of $z$ in particular is entirely determined by $z^{\prime}$ and independent of $\bar{a}$. Moreover $z$ bounds according to (1) if and only if there exists an $x^{\prime} \in C_{*}(X)$ such that $d x^{\prime}=$ $z^{\prime}+a, a \in C_{*}(A)\left(=\left(\right.\right.$ cone $\left.\left.C_{*}(A)\right) \cap C_{*}(A)\right)$. This implies in particular that $H_{*}\left(C_{*}(X, A)\right) \approx$ $h_{*}(X, A) \approx H_{*}\left(K_{*}(X, A)\right)$. The axioms of a chain functor for $K_{*}=\left\{K_{*}, K_{*}^{\prime}, l, i^{\prime}, \varphi, \kappa\right\}$ can be easily verified. The boundary operator $\partial$ defined for $K_{*}$ agrees with that originating from $\S 3$ (1). Since in our case $l=j_{\#} \varphi=j_{\#}, \varphi \kappa=1, \boldsymbol{K}_{*}$ is flat.

Suppose now that $\boldsymbol{K}_{*}$ is a flat chain functor associated with $h_{*}$. Let $C_{*}(X)$ be the mapping cylinder of $\varphi: K_{*}^{\prime}(X, X) \longrightarrow K_{*}(X)$. The only properties of $C_{*}(X)$ we must attend are the existence of natural incluions $K_{*}^{\prime}(X, X), K_{*}(X) \subset C_{*}(X)$, where the second one is a homotopy equivalence, which we are going for simplicity to omit from our notation. $C_{*}: \mathfrak{K} \longrightarrow c h$ is a functor because $\boldsymbol{K}_{*}$ is assumed to be flat. There exists a (in $(X, A)$ ) natural mapping $r: C_{*}(X) \longrightarrow K_{*}(X, A)$ such that for $x \in K_{*}^{\prime}(X, A) \subset C_{*}(X), d x \in i m i_{\#}$ one has $r(x) \sim j_{\#}(x) \sim l(x) \bmod A$ (i.e. everything contained in $A$ remains there).

This helps us calculating the "homology of $C_{*}(X)$ rel. $A$ ":

A cycle rel. $A$ is a chain $z^{\prime}$ in $C_{*}(X)$ satisfying $d z^{\prime} \in i m i_{\#}, i: A \subset X$. Because of the homotopy equivalence $K_{*}(X) \subset C_{*}(X)$, we can assume that $z^{\prime} \in K_{*}(X)$.

We have $z^{\prime} \sim 0$ whenever there are chains $y \in C_{*+1}(X), a \in C_{*}(A)$ such that $d y=z^{\prime}+a$. Again, we can assume that $y \in K_{*+1}, a \in K_{*}(A)$.

This furnishes the homology $H_{*}\left(C_{*}(X) / C_{*}(A)\right)=H_{*}$. There is a natural mapping

$$
\lambda: H_{*} \longrightarrow H_{*}\left(K_{*}(X, A)\right) \approx h_{*}(X, A):
$$

To each $z^{\prime}$ there exists a $\bar{a} \in K_{*}(A, A)$ such that $d \bar{a}=-d z^{\prime}$ (again inclusions omitted from the notation), allowing us to define

$$
\lambda\left[z^{\prime}\right]=\left[z^{\prime}+\bar{a}\right] .
$$

This is well-defined and natural. Because of condition 3) in the definition of a chain functor $(\S 3], \lambda$ is epic.

Suppose that $z^{\prime}+\bar{a} \sim 0$ in $K_{*}(X, A)$, then, according to condition 4) in the definition of a chain functor in $\S 3$, there exists an $a \in K_{*}(A)$ such that $d a=-d z^{\prime}$, so that $z^{\prime}+\bar{a} \sim z^{\prime}+a \sim 0$. Condition 5) ensures that $\kappa\left(z^{\prime}+a\right)-a_{1}=d x, x \in C_{*+1}(X), a_{1} \in C_{*}(A)$.

Application of $\varphi$ yields a $y \in K_{*+1}(X), a_{2} \in K_{*}(A)$ such that $z^{\prime}+a_{2}=d y$, hence $\left[z^{\prime}\right]=0$ in $H_{*}$. Thus $\lambda$ is monic.

Let $z \in Z_{n}\left(K_{*}(X, A)\right)$ be a cycle, then we can assume that $z=z^{\prime}+\bar{a}, \bar{a} \in K_{*}(A, A)$ and $\partial[z]=\left[i_{\#}^{\prime}{ }^{-1} d z^{\prime}\right]=\bar{\partial}\left[z^{\prime}\right]$, where $\bar{\partial}$ is the boundary operator, associated with the short exact sequence (1) in $\S 3$. 
As a result, the short exact sequence (1) in $\S 3$ for this new $C_{*}$ determines the correct homology as well as the correct boundary operator for the given homology theory $h_{*}$.- 


\section{References}

1. D. W. Anderson, Chain functors and homology theories Lecture Notes in Mathem. Vol. 249 (1991) 1 - 12

2. F. W. Bauer, Chain functors with isomorphic homology (preprint)

3. -", Tensor products of spectra and localizations (preprint)

4. -", Colocalizations and their realizations as spectra (preprint)

5. - - , Strong homology theories as localizations (preprint))

6. -"-, Bordism theories and chain complexes Journ. Pure \& Appl. Algebra, 102 (1995) $251-271$

7. -"-, Classifying spectra and generalized homology theories Annali di Mat. pura ed appl. (IV) Vol CLXVI (1993) 365 - 399

8. -"-, Generalized homology theories and chain complexes Annali di Mat. pura ed appl. (IV) Vol CLV (1989) 143 - 191

9. -", Die Homotopiekategorie der Boardman-Spektren ist zur Homotopiekategorie der Kan-Spektren äquivalent Compositio Math. 28 Fasc. 1(1974) 1 - 8

10. -" , Extensions of generalized homology theories Pacific Journal of Math. 128, No. 1, (1987) $25-61$

11. -"-, J.Dugundji, Categorical homotopy and fibrations Trans. Am. Math.Soc. 140 (1969) $239-256$

12. A.K. Bousfield, The localization of spectra with respect to homology Topology, 18 (1979) $257-281$

13. -"-, The Boolean algebra of spectra Comment. Math. Helvetici 54 (1979) 368 - 377

14. S. Buoncristiano, C. Rourke, P. J. Sanderson A geometric approach to homology theory LMS Lecture Notes Ser. 18, London Math. Soc. (1976)

15. R. O. Burdick, P. E. Conner, E. E. Floyd, Chain theories and their derived homology Proceedings of the AMS, 19 (1968) 1115 - 1118

16. F. W. Cathey, Strong shape theory in "Shape theory and Geometric Topology" Lecture Notes in Math. Vol 870 Springer-Verlag (1981) 215 -238

17. A. D. Elmendorf, I. Kriz, M. A. Mandell, J. P. May, Rings, Modules and Algebras in Stable Homotopy Theory Math. Surveys and Monographs 47 AMS, Providence RI (1997)

18. P. Gabriel, M. Zisman, Calculus of Fractions and Homotopy Theory Springer-Verlag, NewYork (1967)

19. A. Heller, Extraordinary homology and chain complexes Proceedings of the Confer. on Categorical Algebra, La Jolla, (1965) 355 - 365

20. P. S. Hirschhorn, Localization of model categories http://www.math.mit.edu/ psh

21. M. Hovey, J. H. Palmieri, N. P. Strickland, Axiomatic stable homotopy theory Mem. Am. Math. Soc. Vol. 128 (1997) No. 610

22. M. Hovey, B. Shipley, J. Smith, Symmetric spectra (1998) (preprint)

23. D. Kan, G. W. Whitehead, The reduced join of two spectra Topology 3 Suppl. 3 (1965) $239-261$

24. H. R. Margolis, "Spectra and the Steenrod Algebra" North-Holland Mathematical Library Vol.29, North-Holland, Amsterdam, NewYork, Oxford (1983)

25. S. O. Kochman, A Chain functor for bordism Trans. Am. Math. Soc. 239 (1978) 167 196

26. A. Kock, L. Kristensen, I. Madsen, Cochain functors for general cohomology theories II Math. Scand. 20 (1967) 151- 176 
This article may be accessed via WWW at http://www.rmi.acnet.ge/hha/ or by anonymous ftp at ftp://ftp.rmi.acnet.ge/pub/hha/volumes/1999/n3/n3.(dvi,ps,dvi.gz,ps.gz)

Friedrich W. Bauer f.w.bauer@mathematik.uni-frankfurt.de

Johann Wolfgang Goethe-Universität

Fachbereich Mathematik

Robert-Mayer Strasse 6-10

60054 Frankfurt a. M.

Germany 